\title{
Bottomonium Suppression in Nucleus-Nucleus Collisions Using Effective Fugacity Quasi-Particle Model
}

\author{
Indrani Nilima and Vineet Kumar Agotiya \\ Centre for Applied Physics, Central University of Jharkhand, Ranchi 835 205, India \\ Correspondence should be addressed to Vineet Kumar Agotiya; agotiya81@gmail.com
}

Received 20 April 2018; Accepted 26 June 2018; Published 12 July 2018

Academic Editor: Chun-Sheng Jia

Copyright ( 2018 Indrani Nilima and Vineet Kumar Agotiya. This is an open access article distributed under the Creative Commons Attribution License, which permits unrestricted use, distribution, and reproduction in any medium, provided the original work is properly cited. The publication of this article was funded by SCOAP ${ }^{3}$.

\begin{abstract}
We have studied the equation of state and dissociation temperature of bottomonium state by correcting the full Cornell potential in isotropic medium by employing the effective fugacity quasi-particle Debye mass. We had also calculated the bottomonium suppression in an expanding, dissipative strongly interacting QGP medium produced in relativistic heavy-ion collisions. Finally we compared our results with experimental data from RHIC $200 \mathrm{GeV} /$ nucleon $\mathrm{Au}-\mathrm{Au}$ collisions, $\mathrm{LHC} 2.76 \mathrm{TeV} / \mathrm{nucleon} \mathrm{Pb}-\mathrm{Pb}$, and LHC 5.02 TeV/nucleon Pb-Pb collisions as a function of number of participants.
\end{abstract}

\section{Introduction}

At the Relativistic Heavy-Ion Collider (RHIC) situated at Brookhaven National Laboratory (BNL), heavy-ion collisions have been studied. After the pioneer work done in the direction of suppression by Matsui and Satz, and some other development of the potential models, suppression was observed by both SPS and RHIC [1]. Due to the Debye screening of the Quantum Chromo-Dynamic (QCD) potential between the two heavy quarks, quarkonia suppression was originally claimed to be an unambiguous signal of the formation of a quark-gluon plasma (QGP). Quarkonia suppression was suggested to be a signature of the QGP and we can measure the suppression ( $\Upsilon$ as well as $J / \psi$ ), both at RHIC and at the LHC.

In heavy-ion collisions to determine the properties of the medium formed in $\mathrm{A}+\mathrm{A}$ collisions and $\mathrm{p}+\mathrm{p}$ collisions, the $\mathrm{A}+\mathrm{A}$ collision deviates from simple superposition of independent $p+p$ collisions. This deviation is quantified with the nuclear modification factor $\left(R_{A A}\right)$. This factor is the ratio of the yield in heavy-ion collisions over the yield in $\mathrm{p}$ $+p$ collisions, scaled by a model of the nuclear geometry of the collision. The value of $R_{A A}=1$ indicates no modification due to the medium. We can say that the probe of interest is suppressed in heavy-ion collisions if $R_{A A}$ is less than 1 .
A quarkonia meson that forms on the outside surface will not dissociate regardless of the temperature of the medium because it does not have a chance to interact with it. This is why we never see a $R_{A A}$ that is equal to zero. The suppression can also be affected by the QGP, the formation time of the quarkonia meson, and the QGP lifetime as well. For instance, a high $p_{T}$ quarkonia meson could have a formation time long enough that it actually does not see the QGP at all and thus is not suppressed.

In the early days most of the interests were focused on the suppression of charmonium states [1-3] of collider experiments at SPS and RHIC, but several observations are yet to be understood; namely, the suppression of $\psi(1 \mathrm{~S})$ does not increase from SPS to RHIC, even though the centreof-mass energy is increased by fifteen times. The heavyion program at the LHC may resolve those puzzles because the beam energy and luminosity are increased by ten times that of the RHIC. Moreover the CMS detector has excellent capabilities for muon detection and provides measurements of $\psi(2 S)$ and the $\Upsilon$ family, which enables the quantitative analysis of quarkonia. That is why the interest may be shifted to the bottomonium states at the LHC energy.

A potential model for the phenomenological descriptions of heavy quarkonium suppression would be quite useful 
inspite of the progress of direct lattice QCD based determinations of the potential. The large mass of heavy quarks and their small relative velocity make the use of nonrelativistic quantum mechanics justifiable to describe the quarkonia in the potential models. This is one of the main goals of this present study that argues for the modification of the full Cornell potential as an appropriate potential for heavy quarkonium at finite temperature. QGP created at RHIC have a very low viscosity to entropy ratio, i.e., $\eta / \mathcal{S} \geq 1 / 4 \pi$ [4-9], and in the nonperturbative domain of QCD, with temperature close to $T_{c}$, the quark matter in the QGP phase is strongly interacting.

In the present paper, we shall employ quasi-particle model for hot QCD equations of state $[10,11]$ to extract the Debye mass [12] which is obtained in terms of quasi-particle degrees of freedom. We first obtained the medium modified heavy quark potential in isotropic medium and estimate the dissociation temperature. Here, we have used the viscous hydrodynamics to define the dynamics of the system created in the heavy-ion collisions. We have included only the shear viscosity and not included the bulk viscosity. We will look the issue of bulk viscosity in near future.

Our work is organized as follows. In Section 2, we briefly discuss our recent work on medium modified potential in isotropic medium. In Sections 2.1 and 2.2 we study the real and imaginary part of the potential in the isotropic medium and effective fugacity quasi-particle model (EQPM) in Section 2.3. In Section 3 we studied binding energy and dissociation temperature of $\Upsilon, \Upsilon^{\prime}$, and $\chi_{b}$ state considering isotropic medium. Using this effective potential and by incorporating quasi-particle Debye mass, we have then developed the equation of state for strongly interacting matter and have shown our results on pressure, energy density, and speed of sound along with the lattice data. In Section 4, we have employed the aforesaid equation of state to study the suppression of bottomonium in the presence of viscous forces and estimate the survival probability in a longitudinally expanding QGP. Results and discussion will be presented in Section 5 and finally, we conclude in Section 6.

\section{Medium Modified Effective Potential in Isotropic Medium}

We can obtain the medium modification to the vacuum potential by correcting its both Coulombic and string part with a dielectric function $\epsilon(p)$ encoding the effect of deconfinement [25]:

$$
V(r, T)=\int \frac{d^{3} \mathbf{p}}{(2 \pi)^{3 / 2}}\left(e^{i \mathbf{p} \cdot \mathbf{r}}-1\right) \frac{V(p)}{\epsilon(p)} .
$$

Here the functions, $\epsilon(p)$ and $V(p)$, are the Fourier transform (FT) of the dielectric permittivity and Cornell potential, respectively. After assuming $r$ as distribution $(r \longrightarrow$ $r \exp (-\gamma r))$ we evaluated the Fourier transform of the linear part $\sigma r \exp (-\gamma r)$ as

$$
-\frac{i}{p \sqrt{2 \pi}}\left(\frac{2}{(\gamma-i p)^{3}}-\frac{2}{(\gamma+i p)^{3}}\right) .
$$

While putting $\gamma=0$, we can write the FT of the linear term $\sigma r$ as

$$
(\widetilde{\sigma r})=-\frac{4 \sigma}{p^{4} \sqrt{2 \pi}} .
$$

Thus the FT of the full Cornell potential becomes

$$
V(p)=-\sqrt{\left(\frac{2}{\pi}\right)} \frac{\alpha}{p^{2}}-\frac{4 \sigma}{\sqrt{2 \pi} p^{4}} .
$$

To obtain the real and imaginary parts of the potential, we put the temporal component of real and imaginary part in terms of retarded (or advanced) and symmetric parts in the Fourier space in isotropic medium which finally gives

$$
\begin{aligned}
& \operatorname{Re} D_{11}^{00}(\omega, p)=\frac{1}{2}\left(D_{R}^{00}+D_{A}^{00}\right), \\
& \operatorname{Im} D_{11}^{00}(\omega, p)=\frac{1}{2} D_{F}^{00} .
\end{aligned}
$$

Let us now discuss the real and imaginary part of the potential modified using the above define $\operatorname{Re} D_{11}^{00}(\omega, p)$ and $\operatorname{Im} D_{11}^{00}(\omega, p)$ along with effective fugacity quasi-particle model (EQPM) in the next subsections.

2.1. Real Part of the Potential in the Isotropic Medium. Now using the real part of retarded (advanced) propagator in isotropic medium, we get

$$
\operatorname{Re} D_{R, A}^{00}(0, p)=-\frac{1}{\left(p^{2}+m_{D}^{2}\right)},
$$

where the real part of the dielectric permittivity (also given in [26-28]) becomes

$$
\epsilon(p)=\left(1+\frac{m_{D}^{2}}{p^{2}}\right) .
$$

Now using (6) and real part of dielectric permittivity (7) in (1), we get

$$
\begin{aligned}
& \operatorname{Re} V_{(i s o)}(r, T) \\
& =\int \frac{d^{3} \mathbf{p}}{(2 \pi)^{3 / 2}}\left(e^{i \mathbf{p} \cdot \mathbf{r}}-1\right)\left(-\sqrt{\left(\frac{2}{\pi}\right)} \frac{\alpha}{p^{2}}-\frac{4 \sigma}{\sqrt{2 \pi} p^{4}}\right) \\
& \quad \times\left(\frac{p^{2}}{\left(p^{2}+m_{D}^{2}\right)}\right)
\end{aligned}
$$

Solving the above integral, we find

$$
\begin{aligned}
\operatorname{Re} V_{(i s o)}(\widehat{s}, T)= & \left(\frac{2 \sigma}{m_{D}}-\alpha m_{D}\right) \frac{e^{-\widehat{s}}}{\widehat{s}}-\frac{2 \sigma}{\widehat{s}}+\frac{2 \sigma}{m_{D}} \\
& -\alpha m_{D},
\end{aligned}
$$

where $\widehat{s}=r m_{D}$. In the limit $\widehat{s} \ll 1$, we have

$$
\operatorname{Re} V_{(i s o)}(\widehat{s}, T) \approx-\frac{2 \sigma}{m_{D} \widehat{s}}-\alpha m_{D}
$$


2.2. Imaginary Part of the Potential in the Isotropic Medium. To obtain the imaginary part of the potential in the QGP medium, the temporal component of the symmetric propagator in the static limit has been considered, which reads $[29,30]$

$$
\operatorname{Im} D_{F(i s o)}^{00}(0, k)=\frac{-2 \pi T m_{D}^{2}}{k\left(k^{2}+m_{D}^{2}\right)^{2}}
$$

Now the imaginary part of the dielectric function in the QGP medium is

$$
\frac{1}{\epsilon(k)}=\pi T m_{D}^{2} \frac{k^{2}}{k\left(k^{2}+m_{D}^{2}\right)^{2}}
$$

Afterwards, the imaginary part of the medium potential is easy to obtain owing to the definition of the potential (1) as done in [31]:

$$
\begin{aligned}
\operatorname{Im} V(r, T)= & -\int \frac{d^{3} \mathbf{k}}{(2 \pi)^{3 / 2}}\left(e^{i \mathbf{k} \cdot \mathbf{r}}-1\right) \\
& \times\left(-\sqrt{\frac{2}{\pi}} \frac{\alpha}{k^{2}}-\frac{4 \sigma}{\sqrt{2 \pi k^{4}}}\right) \frac{-\pi T m_{D}^{2} k}{\left(k^{2}+m_{D}^{2}\right)^{2}}
\end{aligned}
$$

After performing the integration, we find

$$
\operatorname{Im} V_{(i s o)}(\widehat{s}, T)=T\left(\frac{\alpha \widehat{s}^{2}}{3}-\frac{\sigma \widehat{s}^{4}}{30 m_{D}^{2}}\right) \log \left(\frac{1}{\widehat{s}}\right),
$$

where $(\widehat{s})=r m_{D}$.

2.3. Effective Fugacity Quasi-Particle Model (EQPM). In our calculation, we use the Debye mass $m_{D}$ for full QCD:

$$
\begin{aligned}
m_{D}^{2} & =g^{2}(T) T^{2}\left[\left(\frac{N_{c}}{3} \times \frac{6 \text { PolyLog }\left[2, z_{g}\right]}{\pi^{2}}\right)\right. \\
& \left.+\left(\frac{N_{f}}{6} \times \frac{-12 \text { PolyLog }\left[2,-z_{q}\right]}{\pi^{2}}\right)\right] .
\end{aligned}
$$

Here, $g(T)$ is the QCD running coupling constant, $N_{c}=$ $3(S U(3))$ and $N_{f}$ is the number of flavors, the function PolyLog[2,z] has the form PolyLog[2,z] $=\sum_{k=1}^{\infty}\left(z^{k} / k^{2}\right)$, and $z_{g}$ is the quasi-gluon effective fugacity and $z_{q}$ is quasi-quark effective fugacity. These distribution functions are isotropic in nature. These fugacities should not be confused with any conservations law (number conservation) and have merely been introduced to encode all the interaction effects at high temperature QCD. Both $z_{g}$ and $z_{q}$ have a very complicated temperature dependence and asymptotically reach to the ideal value unity [11]. The temperature dependence of $z_{g}$ and $z_{q}$ fits well to the form given below:

$$
z_{g, q}=a_{q, g} \exp \left(-\frac{b_{g, q}}{x^{2}}-\frac{c_{g, q}}{x^{4}}-\frac{d_{g, q}}{x^{6}}\right) .
$$

Here $x=T / T_{c}$ and $a, b, c$, and $d$ are fitting parameters, for both EOS1 and EOS2. Here, EoS1 is the $O\left(g^{5}\right)$ hot QCD [13-15] and EoS2 is the $O\left(g^{6} \ln (1 / g)\right.$ hot QCD EoS [16] in the quasi-particle description $[10,11]$, respectively. Now, the expressions for the Debye mass can be rewritten in terms of effective charges for the quasi-gluons and quarks as

$$
m_{D}^{2}= \begin{cases}Q_{g}^{2} T^{2} \frac{N_{c}}{3} & \text { for pure gauge, } \\ T^{2}\left(\frac{N_{c}}{3} Q_{g}^{2}\right)+\left(\frac{N_{f}}{6} Q_{q}^{2}\right) & \text { for full QCD }\end{cases}
$$

where $Q_{g}$ and $Q_{q}$ are the effective charges given by the equations:

$$
\begin{aligned}
& Q_{g}^{2}=g^{2}(T) \frac{6 \text { PolyLog }\left[2, z_{g}\right]}{\pi^{2}} \\
& Q_{q}^{2}=g^{2}(T) \frac{-12 \text { PolyLog }\left[2,-z_{q}\right]}{\pi^{2}} .
\end{aligned}
$$

In our present analysis we had used the temperature dependence of the quasi-particle Debye mass, $m_{D}^{Q P}$, in full QCD with $N_{f}=3$ to determine charmonium suppression in an expanding, dissipative strongly interacting QGP medium. This quasi-particle Debye mass, $m_{D}^{\mathrm{QP}}$, has the following form:

$$
\begin{aligned}
m_{D}^{\mathrm{QP}} & =\frac{2}{\pi^{2}} g(T) T\left[\frac{N_{c}}{3} \text { PolyLog }\left[2, z_{g}\right]\right. \\
& \left.-N_{f} \text { PolyLog }\left[2,-z_{q}\right]\right]^{1 / 2} .
\end{aligned}
$$

\section{Binding Energy and Dissociation Temperature}

To obtain the binding energies with heavy quark potential, we need to solve the Schrödinger equation numerically. In the limiting case discussed earlier, the medium modified potential resembles to the hydrogen atom problem [1]. The solution of the Schrödinger equation gives the eigenvalues for the ground states and the first excited states in charmonium $\left(J / \psi, \psi^{\prime}\right.$, etc. $)$ and bottomonium $\left(\Upsilon, \Upsilon^{\prime}\right.$, etc. $)$ spectra:

$$
\operatorname{Re}_{\text {bin }}^{\text {iss }} \stackrel{\widehat{s} \gg 1}{=}\left(\frac{\mathrm{m}_{\mathrm{Q}} \sigma^{2}}{\mathrm{~m}_{\mathrm{D}}^{4} \mathrm{n}^{2}}+\alpha \mathrm{m}_{\mathrm{D}}\right) ; \mathrm{n}=1,2 \cdots
$$

where $m_{Q}$ is the mass of the heavy quark.

In our analysis, we have fixed the critical temperature $\left(T_{c}\right.$ $=0.197 \mathrm{GeV}$ ) and have taken the quark masses $m_{\mathrm{Q}}$, as $m_{\Upsilon}=4.5$ $\mathrm{GeV}, m_{\Upsilon^{\prime}}=5.01 \mathrm{GeV}$, and $m_{\chi_{b}}=5.18 \mathrm{GeV}$, as calculated in [32], and the string tension $(\sigma)$ is taken as $0.184 \mathrm{GeV}^{2}$. Let us now proceed to the computation of the dissociation temperatures for the above-mentioned quarkonia bound states.

As we know, dissociation of a quarkonia bound state in a thermal QGP medium will occur whenever the binding energy, $E_{B}$, of the said state will fall below the mean thermal energy of a quasi-parton. In such situations, the thermal effect can dissociate the quakonia bound state. To obtain 
TABLE 1: Dissociation temperature $T_{D}$ (for a 3-flavor QGP), using quasi-particle Debye mass for bottomonium states, for EoS1.

\begin{tabular}{lcccccc}
\hline State & $\tau_{F}$ & $T_{D}$ & $c_{s}^{2}($ SIQGP $)$ & $c_{s}^{2}(\mathrm{Id})$ & $\epsilon_{s}(\mathrm{SIQGP})$ & $\epsilon_{s}(\mathrm{Id})$ \\
\hline$\Upsilon$ & 0.76 & 1.98 & 0.335 & $1 / 3$ & 24.39 & 23.89 \\
\hline$\Upsilon^{\prime}$ & 1.90 & 1.53 & 0.326 & $1 / 3$ & 8.28 & 8.16 \\
\hline$\chi_{b}$ & 2.60 & 1.61 & 0.331 & $1 / 3$ & 10.21 & 10.10 \\
\hline
\end{tabular}

TABLE 2: Dissociation temperature $T_{D}$ (for a 3-flavor QGP), using quasi-particle Debye mass for bottomonium states, for EoS2.

\begin{tabular}{lcccccc}
\hline State & $\tau_{F}$ & $T_{D}$ & $c_{s}^{2}($ SIQGP $)$ & $c_{s}^{2}(\mathrm{Id})$ & $\epsilon_{s}(\mathrm{SIQGP})$ & $\epsilon_{s}(\mathrm{Id})$ \\
\hline$\Upsilon$ & 0.76 & 2.04 & 0.335 & $1 / 3$ & 27.05 & 27.09 \\
\hline$\Upsilon^{\prime}$ & 1.90 & 1.58 & 0.328 & $1 / 3$ & 9.35 & 9.44 \\
\hline$\chi_{b}$ & 2.60 & 1.65 & 0.331 & $1 / 3$ & 11.21 & 11.34 \\
\hline
\end{tabular}

the lower bound of the dissociation temperatures of the various quarkonia states, the (relativistic) thermal energy of the partons will be $3 \mathrm{~T}$. The dissociation is supposed to occur whenever

$$
\operatorname{Re} \mathrm{E}_{\text {bin }}^{\stackrel{\text { iso }}{\stackrel{\widehat{s}}{\Rightarrow}}=1} \mathrm{E}_{\mathrm{B}}\left(\mathrm{T}_{\mathrm{D}}\right)=3 \mathrm{~T}_{\mathrm{D}} .
$$

$T_{D}$ 's for the $b \bar{b}$ sates $\Upsilon, \Upsilon^{\prime}$, and $\chi_{b}$ with the dissociation temperature are listed in Tables 1 and 2 for EoS1 and EoS2, respectively. We observe that (on the basis of temperature dependence of binding energy) $Y^{\prime}$ dissociates at lower temperatures as compared to $\Upsilon$ and $\chi_{b}$ for both the equations of state.

\section{Formulation}

In relativistic nucleus-nucleus collisions, the equation of state for the quark matter is an important observable and the properties of the matter are sensitive to it. The expansion of QGP is quite sensitive to EoS through the speed of sound and explores the sensitivity of the quarkonium suppression to the equation of state $[33,34]$.

For a strongly coupled QGP, Bannur [17] developed an equation of state by incorporating running coupling constant and did an appropriate modification to take account of color and flavor degrees of freedom and obtained a reasonably good fit to the lattice results. Now we will discuss briefly the equation of state which is expressed as a function of plasma parameter $\Gamma[35]$ :

$$
\epsilon_{\mathrm{QED}}=\left(\frac{3}{2}+u_{e x}(\Gamma)\right) n T .
$$

Plasma parameter $\Gamma$ is the ratio of average potential energy to average kinetic energy of particles, is assumed to be weak $(<<1)$, and is given by

$$
\Gamma \equiv \frac{\langle P E\rangle}{\langle K E\rangle}=\frac{\operatorname{Re}[V(\mathbf{r}, T)]}{T} .
$$

We have studied the variation of plasma parameter with temperature and as well with the number of flavors that are present in the system and shown in Figure 1 for EoS1 and EoS2, respectively. As the temperature increases, potential becomes weaker and hence the plasma parameters have started waning; albeit at very large temperature it increases slightly due to the contribution coming from the (positive) finite-range terms in the potential, unlike the decreasing trend in Bannur model [17] always due to the presence of Coulomb interaction alone in the deconfined phase.

Let us consider that hadron exists for $T<T_{c}$ and goes to QGP for $T>T_{c}$ for strongly coupled plasma in QCD. As it was assumed that confinement interactions due to QCD vacuum have been melted [17] at $T=T_{c}$ and thus for $T>T_{c}$, there are the strongly interacting plasma of quarks and gluons and no glue balls or hadrons. After inclusion of relativistic and quantum effects, the equation of state which has been obtained in the plasma parameter can be written as

$$
\varepsilon=\left(3+u_{e x}(\Gamma)\right) n T .
$$

Now, the scaled-energy density is written as in terms of ideal contribution

$$
e(\Gamma) \equiv \frac{\varepsilon}{\varepsilon_{S B}}=1+\frac{1}{3} u_{e x}(\Gamma),
$$

where $\varepsilon_{S B}$ is given by

$$
\varepsilon_{S B} \equiv \frac{\left(16+21 n_{f} / 2\right) \pi^{2} T^{4}}{30} .
$$

Here, $n_{f}$ is the number of flavors of quarks and gluons. Now, we will employ two-loop level QCD running coupling constant in $\overline{\mathrm{MS}}$ scheme [36]:

$$
\begin{aligned}
& g^{2}(T) \\
& \quad \approx 2 b_{0} \ln \frac{\bar{\mu}}{\Lambda_{\overline{\mathrm{MS}}}}\left(1+\frac{b_{1}}{2 b_{0}^{2}} \frac{\ln \left(2 \ln \left(\bar{\mu} / \Lambda_{\overline{\mathrm{MS}}}\right)\right)}{\ln \left(\bar{\mu} / \Lambda_{\overline{\mathrm{MS}}}\right)}\right)^{-1} .
\end{aligned}
$$

Here $b_{0}=\left(33-2 n_{f}\right) /\left(48 \pi^{2}\right)$ and $b_{1}=\left(153-19 n_{f}\right) /\left(384 \pi^{4}\right)$. In $\overline{\mathrm{MS}}$ scheme, $\Lambda \overline{\mathrm{MS}}$ and $\bar{\mu}$ are the renormalization scale and the scale parameter, respectively. For the EoS to depend on the renormalization scale, the physical observables should be scale independent. We invade the problem by trading off the dependence on renormalization scale $\left(\Lambda_{\overline{\mathrm{MS}}}\right)$ to a dependence on the critical temperature $T_{c}$.

$$
\begin{aligned}
\bar{\mu} \exp \left(\gamma_{E}+c\right) & =\Lambda_{\overline{\mathrm{MS}}}(T) \\
\Lambda_{\overline{\mathrm{MS}}}(T) \exp \left(\gamma_{E}+c\right) & =4 \pi \Lambda_{T},
\end{aligned}
$$

where $\gamma_{E}=0.5772156$ and $c=\left(n_{c}-4 n_{f} \ln 4\right) /\left(22 n_{c}-n_{f}\right)$, which is a constant depending on colors and flavors. There are several incertitude, associated with the scale parameter $\bar{\mu}$ and renormalization scale $\Lambda \overline{\mathrm{MS}}$, which occurs in the expression used for the running coupling constant $\alpha_{s}$. This issue has been considered well in literature and resolved by the BLM criterion due to Brodsky, Lepage, and Mackenzie [37]. $\Lambda \overline{\mathrm{MS}}$ is allowed to vary between $\pi T$ and $4 \pi T$ [38]. For our motive, we choose $\Lambda \overline{\mathrm{MS}}$ close to the central value $2 \pi T_{c}$ [39] for $n_{f}=0$ 

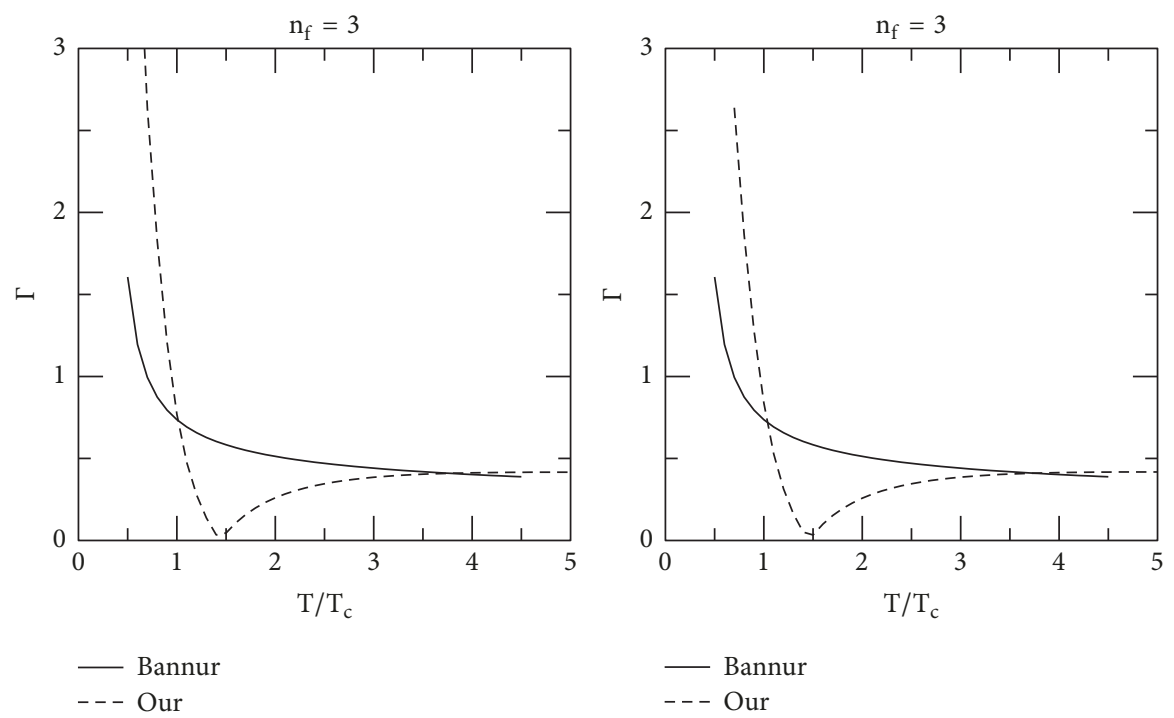

FIGURE 1: Plots of $\Gamma$ as a function of $T / T_{c}$ for 3-flavor QGP (extreme left figure) for EOS1 [13-15] and for EOS2 [16] (extreme right figure). In each figure, solid line represents the results obtained from Bannur EoS, and dashed line represents the results from our EoS (using quasiparticle Debye mass).

and for both $n_{f}=2$ and $n_{f}=3$ flavors the value is $\pi T_{c}$. If the factor $\left(b_{1} / 2 b_{0}^{2}\right)\left(\ln \left(2 \ln \left(\bar{\mu} / \Lambda_{\overline{M S}}\right)\right) / \ln \left(\bar{\mu} / \Lambda_{\overline{\mathrm{MS}}}\right)\right)$ is $\ll 1$, then the above expression reduces to the expression used in [17, Eq. (10)], after neglecting the higher order terms of the above factor. However, this possibility does not hold good for the temperature ranges used in the calculation and causes an error in coupling which finally makes the difference in the results between our model and Bannur model [17]. First of all, we will calculate the energy density $\varepsilon(T)$ from (25) and using the thermodynamic relation

$$
\varepsilon=T \frac{d p}{d T}-P,
$$

we calculated the pressure as

$$
\frac{P}{T^{4}}=\frac{\left(P_{0} / T_{0}+3 a_{f} \int_{T_{0}}^{T} d \tau \tau^{2} e(\Gamma(\tau))\right)}{T^{3}},
$$

where $P_{0}$ is the pressure at some reference temperature $T_{0}$. Now, the speed of sound $c_{s}^{2}(=d P / d \varepsilon)$ can be calculated once we know the pressure $P$ and energy density $\varepsilon$.

\section{Survival of Bottomonium State}

In order to derive the $\Upsilon$ survival probability for an expanding QGP firstly, we explore the effects of dissipative terms up to first order in the stress-tensor. In the presence of viscous forces, the energy-momentum tensor is written as

$$
T^{\mu \nu}-\pi^{\mu \nu}=(\epsilon+p) u^{\mu} u^{\nu}+g^{\mu \nu} p,
$$

where the stress-energy tensor, $\pi^{\mu \nu}$, up to first order is given by

$$
\pi^{\mu \nu}=\eta\left\langle\nabla^{\mu} u^{\nu}\right\rangle
$$

where $\eta$ is the coefficient of the shear viscosity and $\left\langle\nabla^{\mu} u^{\nu}\right\rangle$ is the symmetrized velocity gradient.

In Bjorken expansion, the equation of motion is given by

$$
\partial_{\tau} \epsilon+\frac{\epsilon+p}{\tau}=\frac{4 \eta}{3 \tau^{2}} .
$$

The solution of equation of motion (33) is given as

$$
\begin{aligned}
& \epsilon(\tau) \tau^{\left(1+c_{s}^{2}\right)}+\frac{4 a}{3 \tilde{\tau}^{2}} \tau^{\left(1+c_{s}^{2}\right)}=\epsilon\left(\tau_{i}\right) \tau_{i}^{\left(1+c_{s}^{2}\right)}+\frac{4 a}{3 \widetilde{\tau}_{i}^{2}} \\
& =\text { const, }
\end{aligned}
$$

where the constant is

$$
a=\left(\frac{\eta}{s}\right) T_{i}^{3} \tau_{i}
$$

and the symbols are

$$
\tilde{\tau}^{2}=\left(1-c_{s}^{2}\right) \tau^{2}
$$

and

$$
\widetilde{\tau}_{i}^{2}=\left(1-c_{s}^{2}\right) \tau_{i}^{2}
$$

The first term accounts for the contributions coming from the zeroth-order expansion (ideal fluid) and the second term is the first-order viscous corrections. We now have all the ingredients to write down the survival probability. Chu and Matsui [40] studied the transverse momentum dependence $\left(p_{T}\right)$ of the survival probability by choosing the speed of sound $c_{s}^{2}=1 / 3$ (ideal EoS) and the extreme value $c_{s}^{2}=$ 0 . Instead of taking arbitrary values of $c_{s}^{2}$, we tabulated the values of $c_{s}^{2}$ in Tables 1 and 2 corresponding to the dissociation 
temperatures for bottomonium states for EOS1 and EOS2. One can define initial energy density $\epsilon_{i}$ as

$$
\epsilon_{i}=(1+\beta)\left\langle\epsilon_{i}\right\rangle ; \quad \beta=1
$$

Here, $\beta$ represents the proportionality of the deposited energy to the nuclear thickness where $\left\langle\epsilon_{i}\right\rangle$ is the average initial energy density and will be given by the modified Bjorken formula [41, 42]:

$$
\left\langle\epsilon_{i}\right\rangle=\frac{\xi}{A_{T} \tau_{i}}\left(\frac{d E_{T}}{d y_{h}}\right)_{y_{h}=0},
$$

where $A_{T}$ is the transverse overlap area of the colliding nuclei and $\left(d E_{T} / d y_{h}\right)_{y_{h}=0}$ is the transverse energy deposited per unit rapidity. We use the experimental value of the transverse overlap area $A_{T}$ and the pseudo-rapidity distribution $d E_{T} /\left.d \eta_{h}\right|_{\eta_{h}=0}[43,44]$ at various values of number of participants $N_{\text {part }}$. These $d E_{T} /\left.d \eta_{h}\right|_{\eta_{h}=0}$ numbers are then multiplied by a Jacobian 1.25 to yield the rapidity distribution $d E_{T} /\left.d y_{h}\right|_{y_{h}=0}$ which will be further used to calculate the average initial energy density from Bjorken formula (39). After getting the value of average initial energy density we can obtain the initial energy density from formula (38). The scaling factor $\xi=5$ has been introduced in order to obtain the desired values of initial energy densities $[45,46]$ for most central collision which are consistent with the predictions of the self-screened parton cascade model [47] and also with the requirements of hydrodynamic simulation $[45,46]$ to fit the pseudo-rapidity distribution of charged particle multiplicity $d N_{c h} / d \eta$ for various centralities observed in PHENIX experiments at RHIC energy. Let $\phi$ be the angle between the transverse momentum and position vector $r_{Y}$. Now assuming that $b \bar{b}$ is formed inside screening region at a point whose position vector is $\vec{r}$ and moves with transverse momentum $p_{T}$ making an azimuthal angle, then the condition for escape of $b \bar{b}$ without forming bottomonium states is expressed as

$$
\cos \phi \geq Y ; \quad Y=\frac{\left(r_{s}^{2}-r_{Y}^{2}\right) m-\tau_{F}^{2} p_{T}^{2} / m}{2 r_{\Upsilon} \tau_{F} p_{T}}
$$

where $r_{Y}$ is the position vector at which the bottom, antibottom quark pair is formed, $\tau_{F}$ is the proper formation time required for the formation of bound states of $b \bar{b}$ from correlated $b \bar{b}$ pair, and $m$ is the mass of bottomonia ( $m=M_{\Upsilon}$, $M_{\chi_{b}}, M_{\Upsilon^{\prime}}$ for different resonance states of bottomonium). Assume the radial probability distribution for the production of $b \bar{b}$ pair in hard collisions at transverse distance $r$ as

$$
f(r) \propto\left(1-\frac{r^{2}}{R_{T}^{2}}\right)^{\alpha} \theta\left(R_{T}-r\right) .
$$

Here we take $\alpha=0.5$ in our calculation as used in [40]. Then, in the color screening scenario, the survival probability for the bottomonium in QGP medium can be expressed as [40, 48, 49]

$$
\begin{aligned}
& S\left(p_{T}, N_{\text {part }}\right) \\
& \quad=\frac{2(\alpha+1)}{\pi R_{T}^{2}} \int_{0}^{R_{T}} d r r \phi_{\max }(r)\left\{1-\frac{r^{2}}{R_{T}^{2}}\right\}^{\alpha},
\end{aligned}
$$

where the maximum positive angle $\phi_{\max }$ allowed by (26) becomes [50]

$$
\phi_{\text {max }}(r)= \begin{cases}\pi & \text { if } \mathrm{Y} \leq-1 \\ \pi-\cos ^{-1}|Y| & \text { if } 0 \geq \mathrm{Y} \geq-1 \\ \cos ^{-1}|Y| & 0 \leq \mathrm{Y} \leq-1 \\ 0 & \mathrm{Y} \geq 1\end{cases}
$$

since the experimentalists always measure the quantity, namely, $p_{T}$ integrated nuclear modification factor. We get the theoretical $p_{T}$ integrated survival probability as follows:

$$
S\left(N_{\text {part }}\right)=\frac{\int_{p_{\text {Tmin }}}^{p_{\text {Tmax }}} S\left(p_{T}, N_{\text {part }}\right) d p_{T}}{\int_{p_{\text {Tmin }}}^{p_{T \max }} d p_{T}} .
$$

In nucleus-nucleus collisions, it is known that only about $60 \%$ of the observed $\Upsilon$ originate directly in hard collisions while $30 \%$ of them come from the decay of $\chi_{b}$ and $10 \%$ from the decay of $Y^{\prime}$. Hence, the $p_{T}$-integrated inclusive survival probability of $\Upsilon$ in the QGP becomes $[33,51]$

$$
\left\langle S^{\text {incl }}\right\rangle=0.6\left\langle S^{\text {dir }}\right\rangle_{\Upsilon}+0.3\left\langle S^{\text {dir }}\right\rangle_{\chi_{b}}+0.1\left\langle S^{\text {dir }}\right\rangle_{\Upsilon^{\prime}}
$$

\section{Results and Discussions}

In our results, we had obtained the variation of plasma parameter with temperature and as well with the number of flavors that are present in the system and shown in Figure 1 for EoS1 and EoS2, respectively. After that, in Figure 2, we have plotted the variation of pressure $\left(P / T^{4}\right)$ with temperature $\left(T / T_{c}\right)$ using EoS1 and EoS2 for 3-flavor QGP along with Bannur EoS [17] and compared it with lattice results [1721]. For each flavor, $g_{c}$ and $\Lambda_{T}$ are adjusted to get a good fit to lattice results in Bannur model. Now, energy density $\varepsilon$, speed of sound $c_{s}^{2}$, and so forth can be derived since we had obtained the pressure, $P(T)$. In Figure 3, we had plotted the energy density $\left(\varepsilon / T^{4}\right)$ with temperature $\left(T / T_{c}\right)$ using EoS1 [13-15] and EoS2 for 3-flavor QGP along with Bannur EoS [17] and compared it with lattice result [17-21]. In Figure 4, the speed of sound, $c_{s}^{2}$, is plotted using EoS1 and EoS2 for 3flavor QGP along with Bannur EoS [17]. Since lattice results are not available for 3 flavors, therefore comparison has not been checked for the above-mentioned flavor. Our flavored results match excellent with the lattice results.

In this paper, we had calculated the dissociation temperatures for the bottomonium states $\left(\Upsilon, \Upsilon^{\prime}, \chi_{b}\right.$, etc.), by modifying the Cornell potential and incorporating the quasiparticle Debye mass. On that dissociation temperature, we 

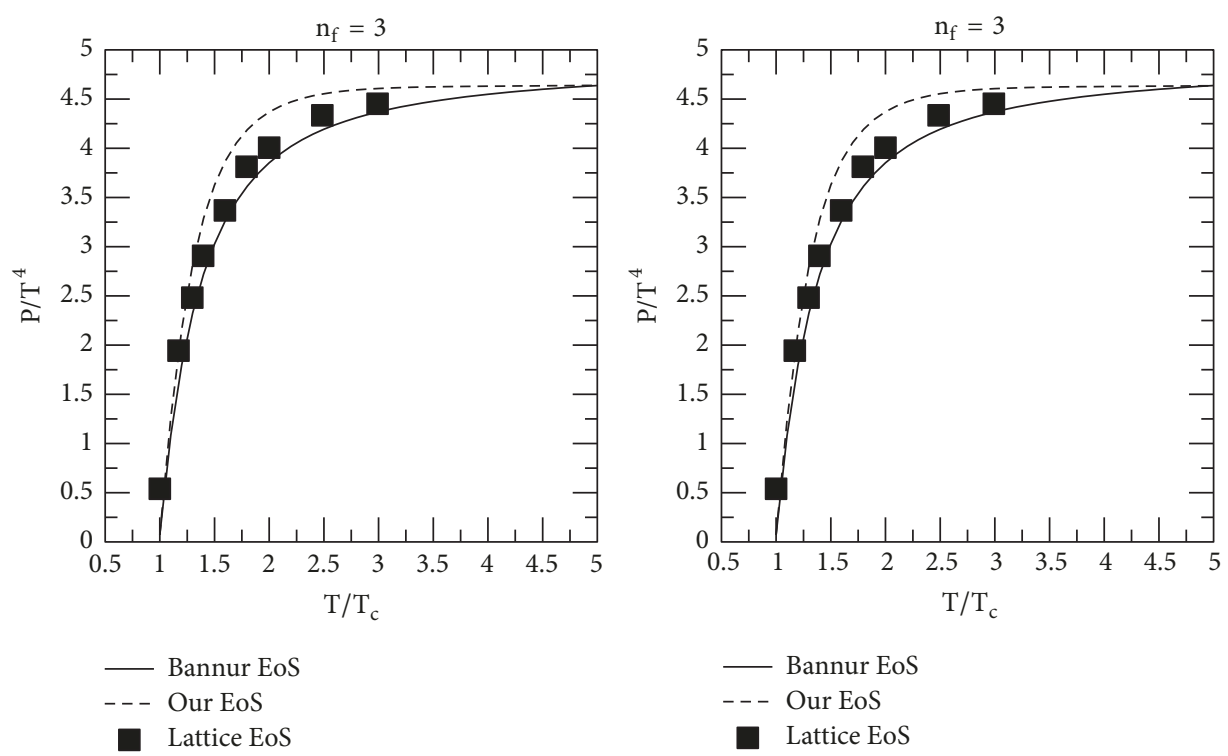

FIgURE 2: Plots of $P / T^{4}$ as a function of $T / T_{c}$ for 3-flavor QGP (extreme left figure) for EOS1 [13-15] and for EOS2 [16] (extreme right figure). In each figure, solid line represents the results obtained from Bannur EoS, dashed line represents the results from our EoS, and diamond symbols represent lattice results [17-21].
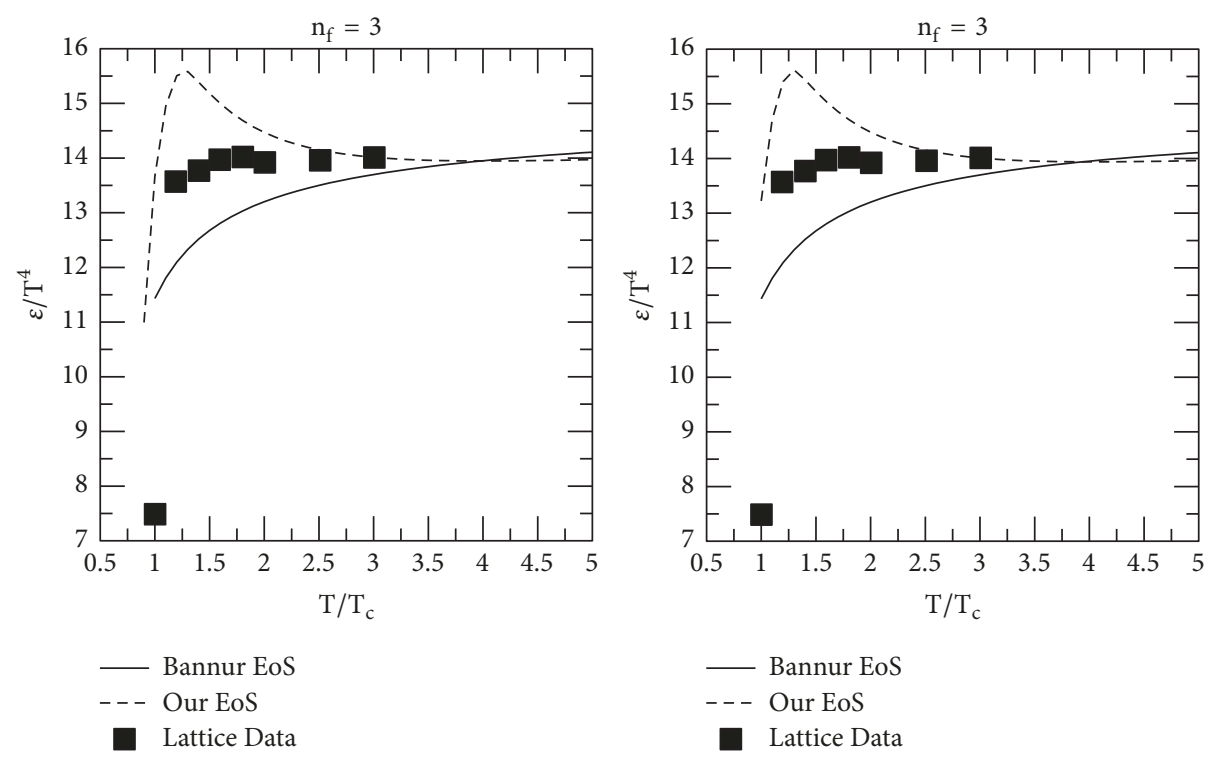

FIgURE 3: Plots of $\varepsilon / T^{4}$ as a function of $T / T_{c}$ for our EoS (using quasi-particle Debye mass) and lattice results [17-21] for 3-flavor QGP (extreme left figure) for EoS1 [13-15] and for EOS2 [16] (extreme right figure). The notations are the same as Figure 2.

had calculated the screening energy densities, $\epsilon_{s}$, and the speed of sound $c_{s}^{2}$ which are also listed in Tables 1 and 2 for both EoS1 and EoS2, respectively. We observe from Tables 1 and 2 that the value of $\epsilon_{s}$ is different for different bottomonium states and varies from one EoS to other. If $\epsilon_{s} \gtrsim$ $\epsilon_{i}$, initial energy density, then there will be no suppression at all, i.e., survival probability, $S\left(p_{T}\right)$, is equal to 1 . With this physical understanding, we analyze our results, $\left\langle S\left(p_{T}\right)\right\rangle$, as a function of the number of participants $N_{\text {Part }}$ in an expanding QGP.
Here we are using the values as inputs listed in Tables 1 and 2, to calculate $\left\langle S\left(p_{T}\right)\right\rangle$ for both EOS1 and EOS2, respectively. The experimental data (the nuclear modification factor $R_{A A}$ ) are shown by the squares with error bars whereas circles represent sequential suppression. We had compared our results with the experimental results for the case of $\eta / s=$ 0.08 for both EoS1 and EoS2 and found good agreement. We observe from Figures 5-10 that $\left\langle S\left(p_{T}\right)\right\rangle$ for both the directly and sequentially produced Upsilon $(\Upsilon)$ are quite high with the higher values of $T_{D}$ 's which is obtained from EOS2 (in 

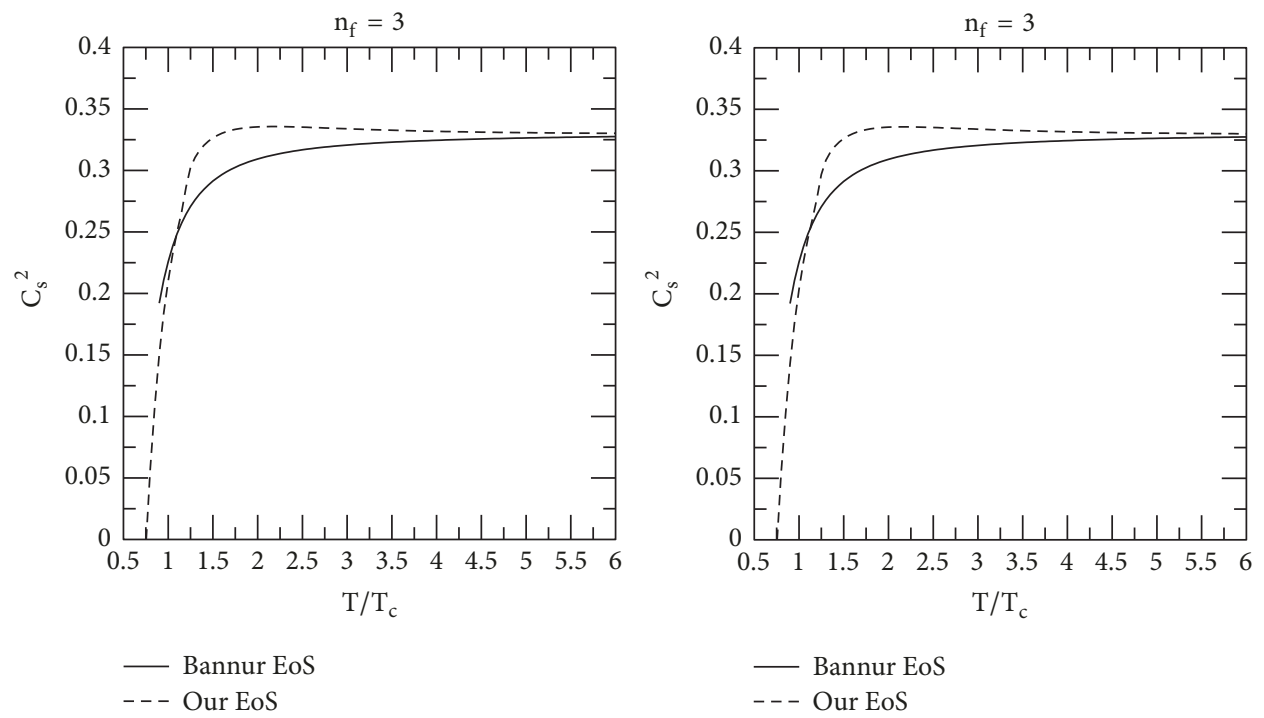

FIGURE 4: Plots of $c_{s}^{2}$ as a function of $T / T_{c}$ for Bannur EoS, our EoS (using quasi-particle Debye mass) for 3-flavor QGP (extreme left figure) for EoS1 [13-15] and for EOS2 [16] (extreme right figure). The notations are the same as Figure 2.
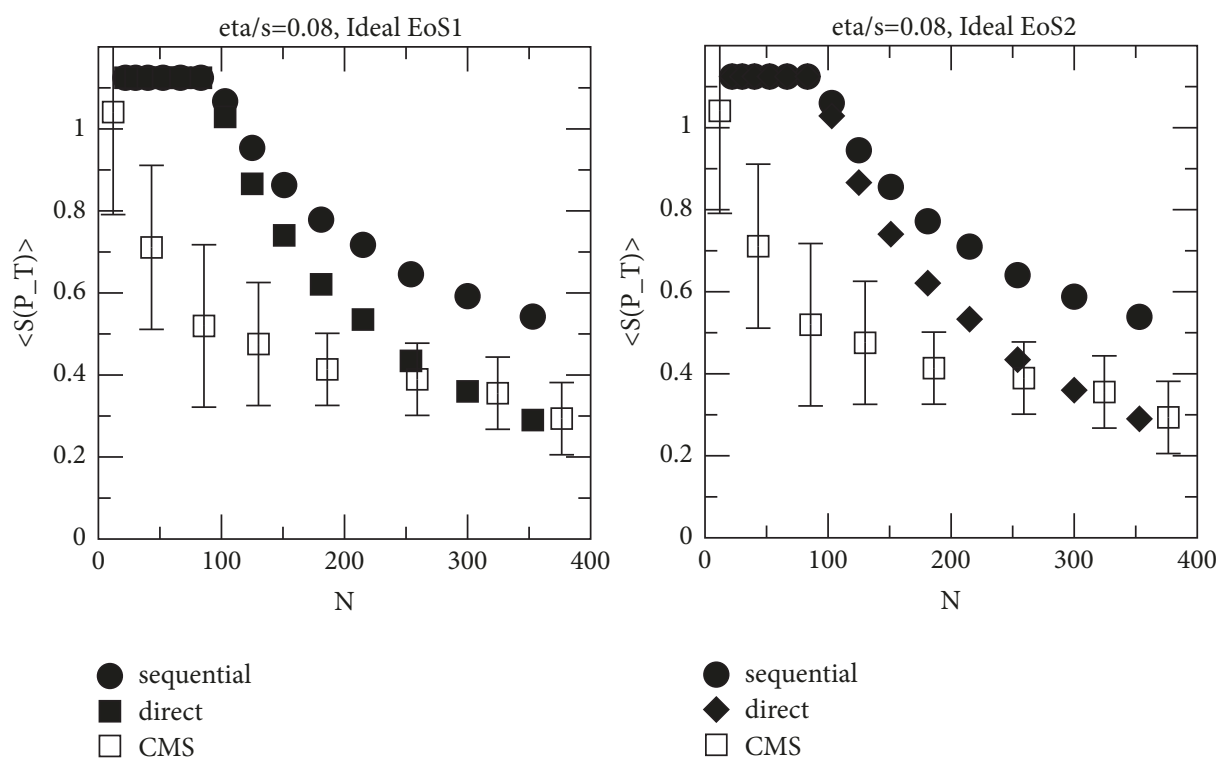

Figure 5: The variation of $p_{T}$ integrated survival probability versus $N$ for $\Upsilon$ at $\sqrt{S_{N N}}=2.76 \mathrm{TeV}$ with preliminary CMS data [22]. The experimental data are shown by the squares with error bars whereas circles and diamond represent $\left(\left\langle S^{\text {incl }}\right\rangle\right)$ without $\left(\left\langle S^{\text {dir }}\right\rangle\right)$ sequential melting using the value of $T_{D}$ 's and related parameters from Tables 1 and 2 for ideal equation of state. Left panel shows EoS1 and right panel shows EoS2.

Table 2) compared to EOS1 (in Table 1) for both SIQGP and ideal equation of states. We find that the survival probability of sequentially produced $\Upsilon$ is slightly higher compared to the directly produced $\Upsilon$ and is closer to the experimental results. We also observed that sequentially produced $\Upsilon$ nicely matches for the EOS1 compared to the EOS2. The smaller value of screening energy density $\epsilon_{s}$ causes an increase in the screening time and results in more suppression to match with the experimental results.

\section{Conclusions}

We studied the equation of state for strongly interacting quark-gluon plasma in the framework of strongly coupled plasma with appropriate modifications to take account of color and flavor degrees of freedom and QCD running coupling constant. In addition, we incorporate the nonperturbative effects in terms of nonzero string tension in the deconfined phase, unlike the Coulomb interactions alone 

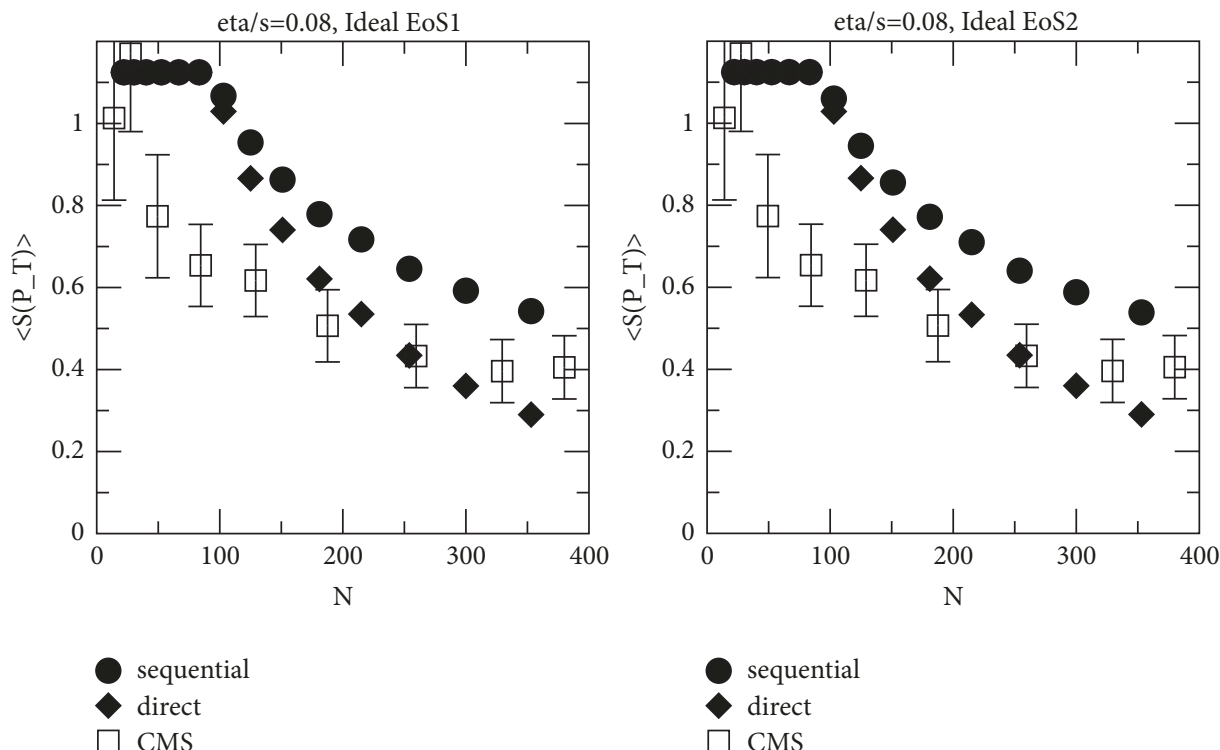

Figure 6: Same as Figure 5 but the variation of $p_{T}$ integrated survival probability versus $N$ for $\Upsilon$ at $\sqrt{S_{N N}}=5.02 \mathrm{TeV}$ with preliminary CMS data [23].
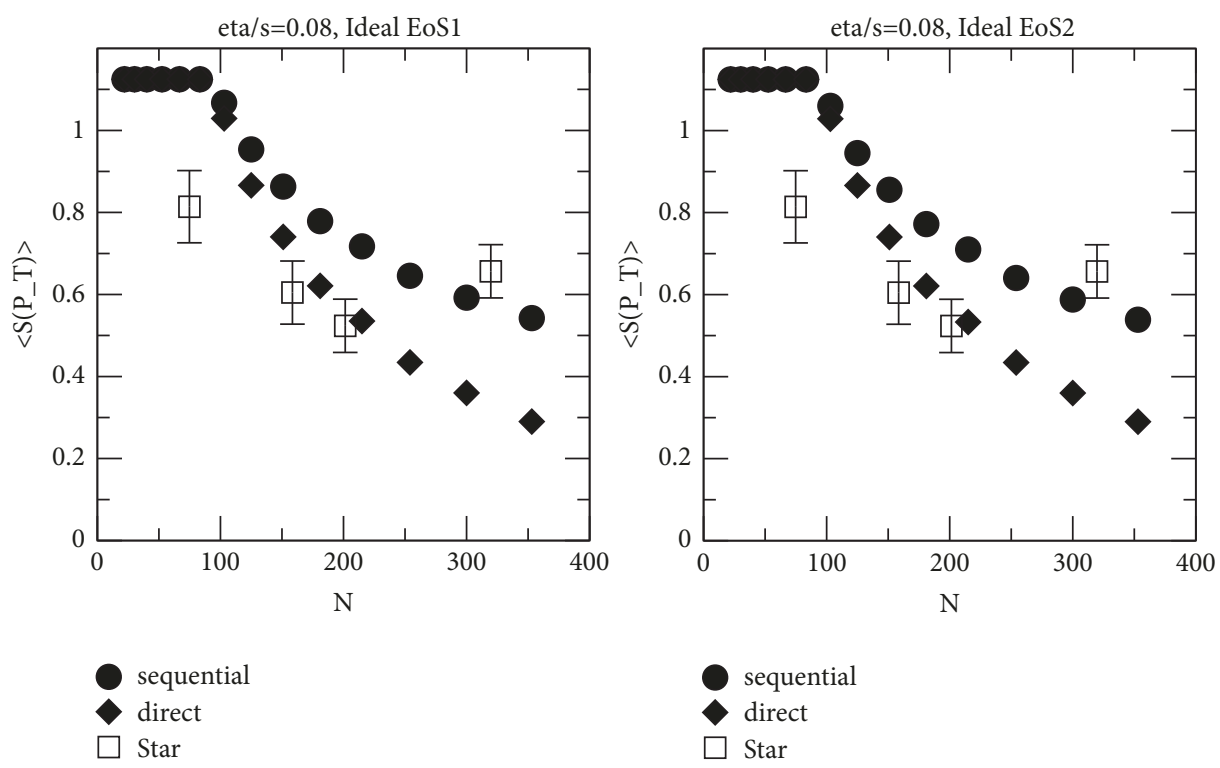

FIGURE 7: Same as Figure 5 but the variation of $p_{T}$ integrated survival probability versus $N$ for $\Upsilon$ at $\sqrt{S_{N N}}=200 \mathrm{GeV}$ with preliminary STAR data [24].

in the deconfined phase beyond the critical temperature. Our results on thermodynamic observables, namely, pressure, energy density, and speed of sound, nicely fit the results of lattice equation of state. We had then calculated the dissociation temperatures for the bottomonium states $\left(\Upsilon, \Upsilon^{\prime}, \chi_{b}\right.$, etc.), by incorporating the quasi-particle Debye mass. On that dissociation temperature, we had calculated the screening energy densities, $\epsilon_{s}$, and the speed of sound $c_{s}^{2}$ which are listed in Tables 1 and 2 for both EoS1 and EoS2, respectively. By using the above quantities as an input, we have then studied the sequential suppression for bottomonium states at the LHC energy in a longitudinally expanding partonic system, which underwent through the successive preequilibrium and equilibrium phases in the presence of dissipative forces. Bottomonium suppression in nucleus-nucleus collisions compared to $p$ - $p$ collisions couples the in-medium properties of the bottomonia states with the dynamics of the expanding medium. We have found a good agreement with the experimental data from RHIC $200 \mathrm{GeV} /$ nucleon $\mathrm{Au}-$ $\mathrm{Au}$ collisions, LHC $2.76 \mathrm{TeV} /$ nucleon $\mathrm{Pb}-\mathrm{Pb}$, and LHC 5.02 $\mathrm{TeV} /$ nucleon $\mathrm{Pb}-\mathrm{Pb}$ collisions $[52,53]$. Here our attempt is to understand $\Upsilon$ suppression systematically in SIQGP in 

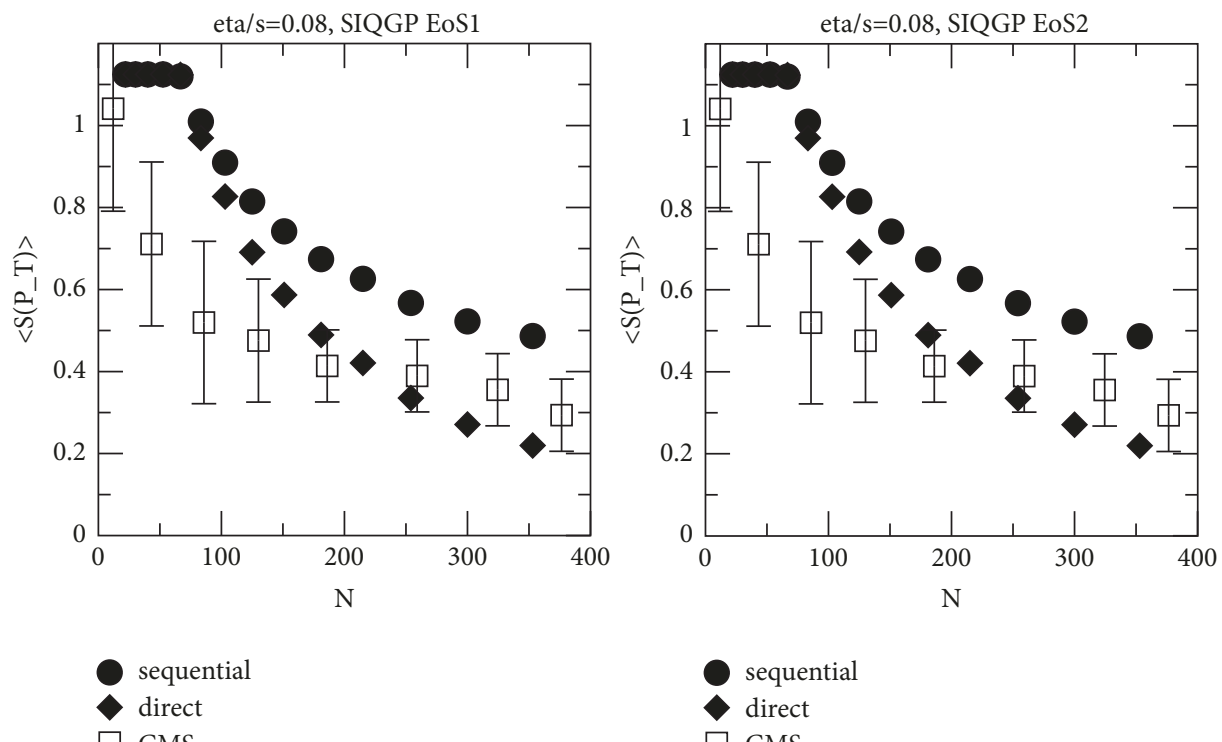

Figure 8: The variation of $p_{T}$ integrated survival probability versus $N$ for $\Upsilon$ at $\sqrt{S_{N N}}=2.76 \mathrm{TeV}$ with preliminary CMS data [22]. The experimental data are shown by the squares with error bars whereas circles and diamond represent $\left(\left\langle S^{\text {incl }}\right\rangle\right)$ without $\left(\left\langle S^{\text {dir }}\right\rangle\right)$ sequential melting using the value of $T_{D}$ 's and related parameters from Tables 1 and 2 for SIQGP equation of state. Left panel shows EoS1 and right panel shows EoS2.
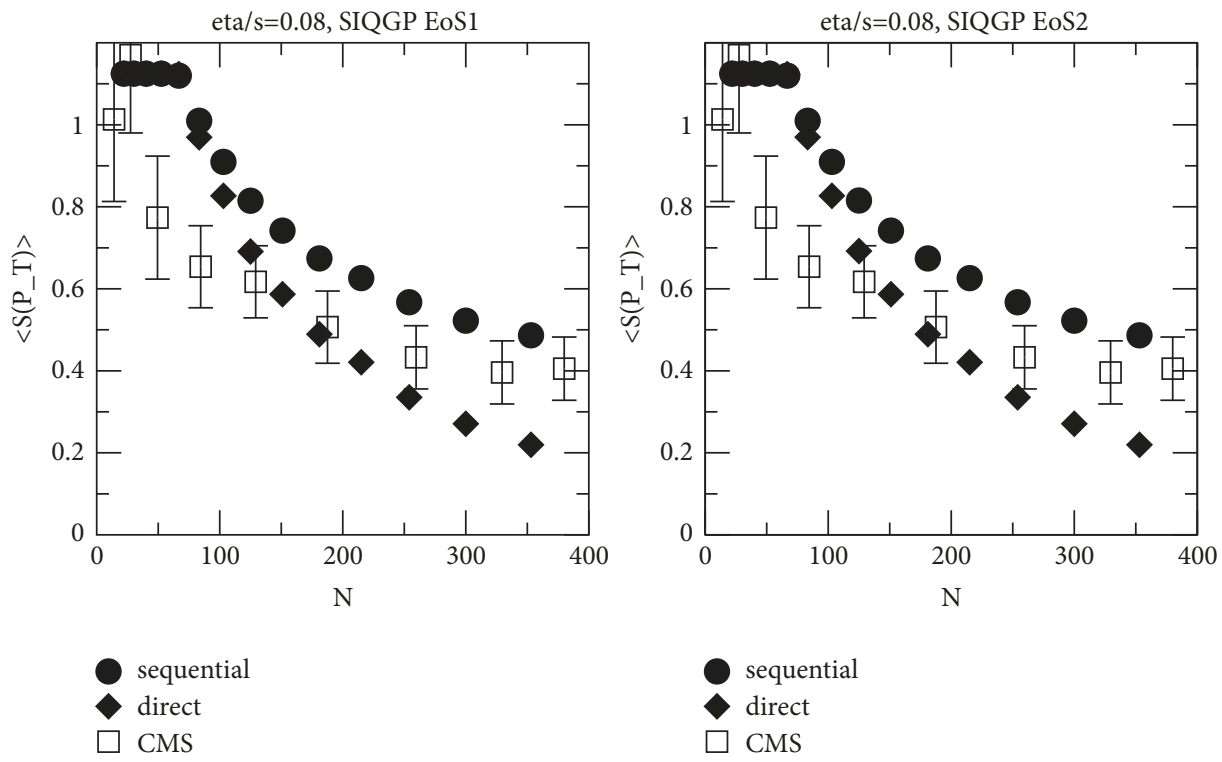

FIGURE 9: Same as Figure 8 but the variation of $p_{T}$ integrated survival probability versus $N$ for $\Upsilon$ at $\sqrt{S_{N N}}=5.02 \mathrm{TeV}$ with preliminary CMS data [23].

anisotropic medium. It would be of interest to extend the present study by incorporating the contributions of the bulk viscosity. These issues will be taken up separately in the near future.

\section{Data Availability}

The data used to support the findings of this study are available from the corresponding author upon request.

\section{Conflicts of Interest}

The authors declare that they have no conflicts of interest.

\section{Acknowledgments}

Vineet Kumar Agotiya acknowledges the UGC-BSR research start up Grant no. F.30-14/2014 (BSR), New Delhi. The authors record their sincere gratitude to the people of India for their generous support for the research in basic sciences. 

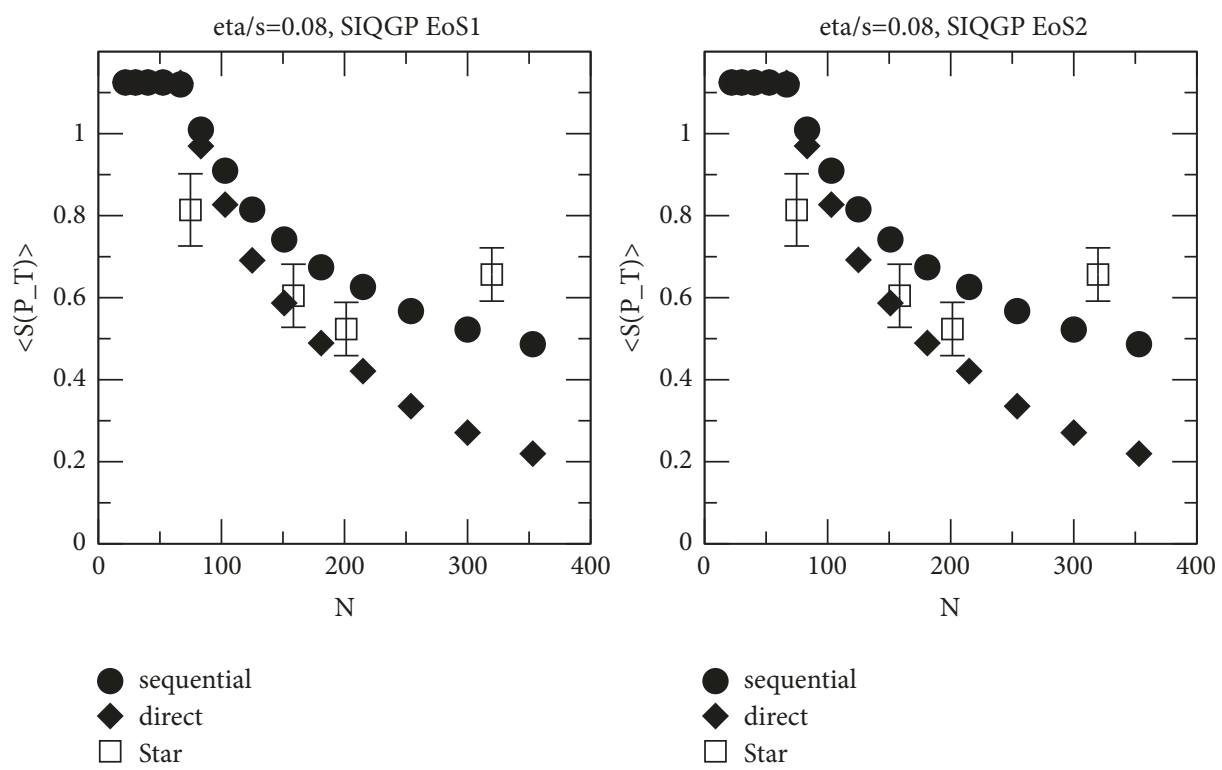

FIGURE 10: Same as Figure 8 but the variation of $p_{T}$ integrated survival probability versus $N$ for $\Upsilon$ at $\sqrt{S_{N N}}=200 \mathrm{GeV}$ with preliminary STAR data [24].

\section{References}

[1] T. Matsui and H. Satz, "J/ $\psi$ suppression by quark-gluon plasma formation," Physics Letters B, vol. 178, no. 4, pp. 416-422, 1986.

[2] F. Karsch, M. T. Mehr, and H. Satz, "Color screening and deconfinement for bound states of heavy quarks," Zeitschrift für Physik C Particles and Fields, vol. 37, no. 4, pp. 617-622, 1988.

[3] F. Karsch and H. Satz, "The spectral analysis of strongly interacting matter," Zeitschrift für Physik C: Particles and Fields, vol. 51, no. 2, pp. 209-224, 1991.

[4] STAR Collaboration, J. Adams, M. M. Aggarwal et al., "Experimental and theoretical challenges in the search for the quark-gluon plasma: The STAR Collaboration's critical assessment of the evidence from RHIC collisions," Nuclear Physics A, vol. 757, no. 1-2, pp. 102-183, 2005.

[5] PHENIX Collaboration, K. Adcox, S. S. Adler et al., "Formation of dense partonic matter in relativistic nucleus-nucleus collisions at RHIC: Experimental evaluation by the PHENIX Collaboration," Nuclear Physics A, vol. 757, no. 1-2, pp. 184-283, 2005.

[6] B. B. Back, M. D. Baker, and M. Ballintijn, "The PHOBOS perspective on discoveries at RHIC," Nuclear Physics A, vol. 757, no. 1-2, pp. 28-101, 2005.

[7] H.-J. Drescher, A. Dumitru, C. Gombeaud, and J.-Y. Ollitrault, "Centrality dependence of elliptic flow, the hydrodynamic limit, and the viscosity of hot QCD," Physical Review C: Nuclear Physics, vol. 76, no. 2, Article ID 024905, 2007.

[8] E. Shuryak, "Toward the theory of strongly coupled quarkgluon plasma," Nuclear Physics A, vol. 774, pp. 387-396, 2006.

[9] P. K. Kovtun, D. T. Son, and A. O. Starinets, "Viscosity in strongly interacting quantum field theories from black hole physics," Physical Review Letters, vol. 94, no. 11, Article ID 111601, 2005.

[10] V. Chandra, A. Ranjan, and V. Ravishankar, "On the chromoelectric permittivity and Debye screening in hot QCD," European Physical Journal A, vol. 40, no. 1, pp. 109-117, 2009.
[11] V. Chandra, R. Kumar, and V. Ravishankar, "Hot QCD equations of state and relativistic heavy ion collisions," Physical Review C, vol. 76, no. 6, Article ID 054909, 2007.

[12] V. Chandra and V. Ravishankar, "Quasi-particle model for lattice QCD: quark-gluon plasma in heavy ion collisions," The European Physical Journal C, vol. 64, no. 1, pp. 63-72, 2009.

[13] C. Zhai and B. Kastening, "Free energy of hot gauge theories with fermions through," Physical Review D: Particles, Fields, Gravitation and Cosmology, vol. 52, no. 12, pp. 7232-7246, 1995.

[14] P. Arnold and C. Zhai, "Three-loop free energy for pure gauge QCD," Physical Review D: Particles, Fields, Gravitation and Cosmology, vol. 50, no. 12, pp. 7603-7623, 1994.

[15] P. Arnold and C. Zhai, "Three-loop free energy for hightemperature QED and QCD with fermions," Physical Review D: Particles, Fields, Gravitation and Cosmology, vol. 51, no. 4, pp. 1906-1918, 1995.

[16] K. Kajantie, M. Laine, K. Rummukainen, and Y. Schröder, "Pressure of hot QCD up to," Physical Review D: Particles, Fields, Gravitation and Cosmology, vol. 67, no. 10, Article ID 105008, 2003.

[17] V. M. Bannur, "Strongly coupled quark gluon plasma (SCQGP)," Journal of Physics G: Nuclear and Particle Physics, vol. 32, no. 7, article 993, 2006.

[18] G. Boyd, J. Engels, and F. Karsch, "Equation of state for the SU(3) gauge theory," Physical Review Letters, vol. 75, no. 23, pp. 41694172, 1995.

[19] G. Boyd, J. Engels, F. Karsch et al., "Thermodynamics of SU(3) lattice gauge theory," Nuclear Physics B, vol. 469, no. 3, pp. 419444, 1996.

[20] F. Karsch et al., "Lattice QCD at high temperature and density," Lecture Notes in Physics, vol. 583, pp. 209-249, 2002, https:// arxiv.org/abs/hep-lat/0106019.

[21] A. Bazavov, T. Bhattacharya, M. Cheng et al., "Equation of state and QCD transition at finite temperature," Physical Review Journals, vol. 80, Article ID 014504, 2009, https://arxiv.org/abs/0903 .4379 . 
[22] CMS Collaboration Twiki, CMS-PAS-HIN-10-006, 2015.

[23] Chad Flores (ALICE Collaboration), "Quark matter," 2017, https://goo.gl/imTtmM.

[24] Ye. Zaochen (STAR Collaboration, URL https, https://goo.gl/ XgqSgGQuark matter 2017.

[25] V. Agotiya, V. Chandra, and B. K. Patra, "Dissociation of quarkonium in a hot QCD medium: modification of the interquark potential," Physical Review C: Nuclear Physics, vol. 80, no. 2, Article ID 025210, 2009.

[26] R. A. Schneider, "Debye screening at finite temperature reexamined," Physical Review D, vol. 66, Article ID 036003, 2002.

[27] H. A. Weldon, "Covariant calculations at finite temperature: the relativistic plasma," Physical Review D: Particles, Fields, Gravitation and Cosmology, vol. 26, no. 6, pp. 1394-1407, 1982.

[28] J. I. Kapusta and C. Gale, Finite Temperature Field Theory Principle and Applications, Cambridge University Press, Cambridge, UK, 2nd edition, 1996.

[29] Y. Burnier, M. Laine, and M. Vepsäläinen, "Quarkonium dissociation in the presence of a small momentum space anisotropy," Physics Letters B, vol. 678, no. 1, pp. 86-89, 2009.

[30] V. K. Agotiya, V. Chandra, M. Y. Jamal, and I. Nilima, "Dissociation of heavy quarkonium in hot QCD medium in a quasiparticle model," Physical Review D: Particles, Fields, Gravitation and Cosmology, vol. 94, no. 9, Article ID 094006, 2016.

[31] L. Thakur, U. Kakade, and B. K. Patra, "Dissociation of quarkonium in a complex potential," Physical Review D: Particles, Fields, Gravitation and Cosmology, vol. 89, no. 9, Article ID 094020, 2014.

[32] V. M. Aulchenko, KEDR Collaboration et al., "New precision measurement of the J/ $\psi$ - and $\psi 1$-meson masses," Physics Letters $B$, vol. 573, pp. 63-79, 2003.

[33] D. Pal, B. K. Patra, and D. K. Srivastava, "Determination of the equation of state of quark matter from $J / \psi$ and $\Upsilon$ suppression at RHIC and LHC," The European Physical Journal C, vol. 17, pp. 179-186, 2000.

[34] B. K. Patra and D. K. Srivastava, " $J / \psi$ suppression: gluonic dissociation vs. colour screening," Physics Letters B, vol. 505, no. 1-4, pp. 113-118, 2001.

[35] S. Ichimaru, "Statistical plasma physics," in Condensed Plasma, vol. 2, Addison-Wesley Publishing Company, New York, NY, USA, 1994.

[36] M. Laine and Y. Schroder, "Two-loop QCD gauge coupling at high temperatures," Journal of High Energy Physics, vol. 2005, no. 03, pp. 067-067, 2005.

[37] S. Huang and M. Lissia, "The relevant scale parameter in the high temperature phase of QCD," Nuclear Physics B, vol. 438, no. 1-2, pp. 54-66, 1995.

[38] E. Braaten and A. Nieto, "Free energy of QCD at high temperature," Physical Review D: Particles, Fields, Gravitation and Cosmology, vol. 53, no. 6, pp. 3421-3437, 1996.

[39] A. Vuorinen, "The pressure of QCD at finite temperature and quark number density," 2004, https://arxiv.org/abs/hep-ph/ 0402242.

[40] M. Chu and T. Matsui, "Pattern of $J / \psi$ suppression in ultrarelativistic heavy-ion collisions," Physical Review D: Particles, Fields, Gravitation and Cosmology, vol. 37, no. 7, pp. 1851-1855, 1988.

[41] B. Alessandro, C. Alexa, and R. Arnaldi, "A new measurement of $J / \psi$ suppression in $\mathrm{Pb}-\mathrm{Pb}$ collisions at $158 \mathrm{GeV}$ per nucleon," The European Physical Journal C, vol. 39, no. 3, pp. 335-345, 2005.
[42] F. Karsch, D. Kharzeev, and H. Satz, "Sequential charmonium dissociation,” Physics Letters B, vol. 637, no. 1-2, pp. 75-80, 2006.

[43] S. S. Adler, (PHENIX Collaboration) et al., "Systematic studies of the centrality and $\sqrt{S_{N N}}$ dependence of the $d E_{T} / d \eta$ and $d N_{\mathrm{ch}} / d \eta$ in heavy ion collisions at midrapidity," Physical Review C, vol. 71, Article ID 034908, 2005.

[44] S. S. Adler, (PHENIX Collaboration) et al., "Systematic studies of the centrality and $\sqrt{S_{N N}}$ dependence of the $d E_{T} / d \eta$ and $d N_{\mathrm{ch}} / d \eta$ in heavy ion collisions at midrapidity," Physical Review C, vol. 71, Article ID 049901(E), 2005.

[45] T. Hirano, "Is early thermalization achieved only near midrapidity in $\mathrm{Au}+\mathrm{Au}$ collisions at $\sqrt{S_{N N}}=130 \mathrm{GeV}$ ?" Physical Review C, vol. 65, Article ID 011901, 2001.

[46] T. Hirano and K. Tsuda, "Collective flow and two-pion correlations from a relativistic hydrodynamic model with early chemical freeze-out," Physical Review C: Nuclear Physics, vol. 66, no. 5, Article ID 054905, 2002.

[47] K. J. Eskola, K. Kajantie, P. V. Ruuskanen, and K. Tuominen, "Scaling of transverse energies and multiplicities with atomic number and energy in ultrarelativistic nuclear collisions," Nuclear Physics, vol. 570, pp. 379-389, 2000.

[48] M. Mishra, C. P. Singh, V. J. Menon, and R. K. Dubey, "J/ $\psi$ suppression in $\mathrm{Au}+\mathrm{Au}$ collisions at RHIC: colour screening scenario in the bag model at variable participant numbers," Physics Letters B, vol. 656, pp. 45-50, 2007.

[49] M. Mishra, C. P. Singh, and V. J. Menon, "J/ $\psi$ suppression vs centrality at forward and mid-rapidity in $\mathrm{Au}+\mathrm{Au}$ collisions at RHIC in colour screening mechanism," Indian Journal of Physics, vol. 85, no. 6, pp. 849-853, 2011.

[50] V. Agotiya, L. Devi, U. Kakade, and B. K. Patra, "Strongly interacting QGP and quarkonium suppression at rhic and LHC energies," International Journal of Modern Physics A, vol. 27, no. 2, 2012.

[51] H. Satz, "Quarkonium binding and dissociation: the spectral analysis of the QGP," Nuclear Physics A, vol. 783, pp. 249-260, 2007, https://arxiv.org/abs/hep-ph/0609197.

[52] B. Krouppa, R. Ryblewski, and M. Strickland, "Bottomonium suppression in heavy-ion collisions," Nuclear Physics A, vol. 967, pp. 604-607, 2017.

[53] B. Krouppa, R. Ryblewski, and M. Strickland, "Bottomonia suppression in $2.76 \mathrm{TeV} \mathrm{Pb}-\mathrm{Pb}$ collisions," Physical Review C, vol. 92, Article ID 061901, 2015. 

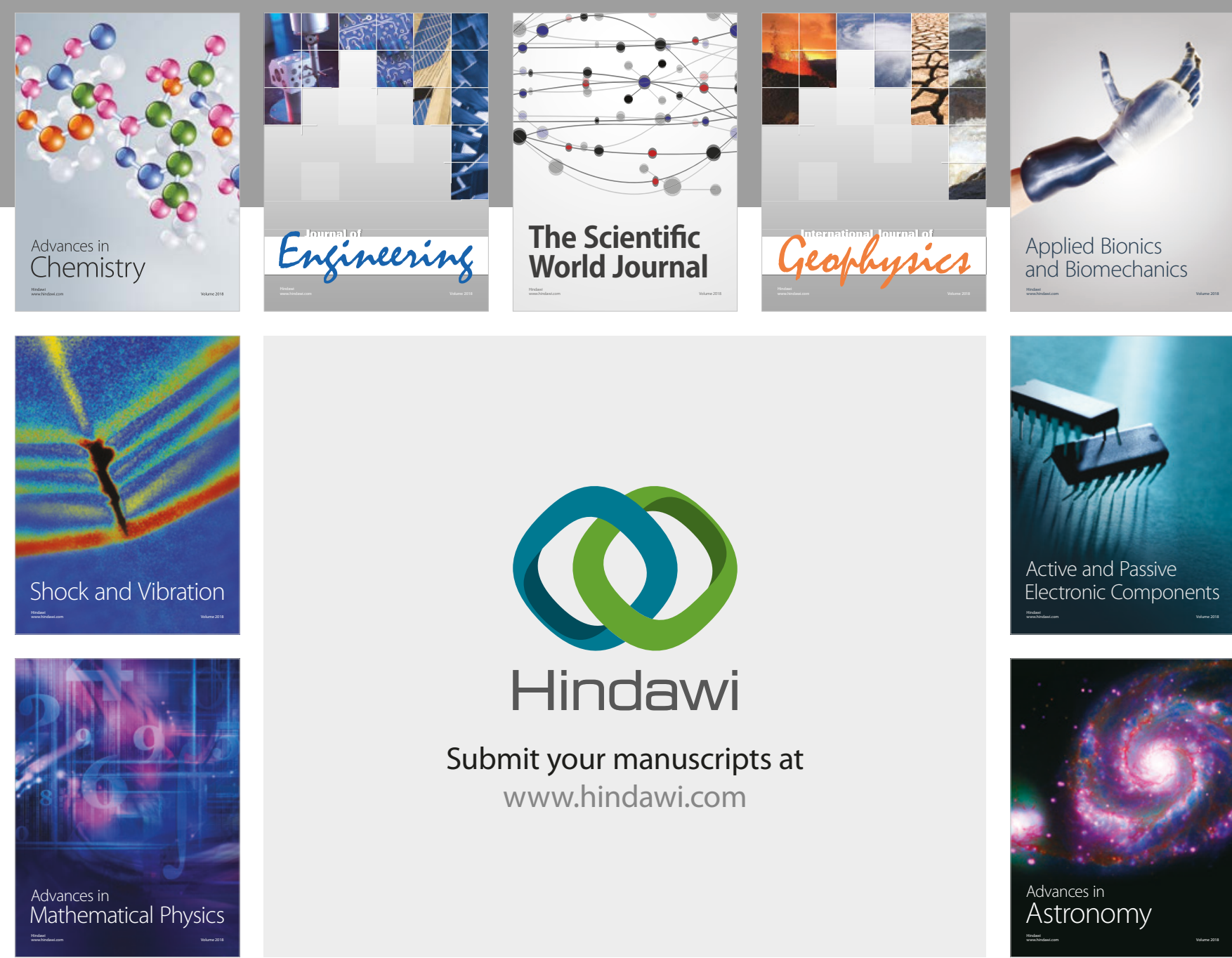

Submit your manuscripts at

www.hindawi.com

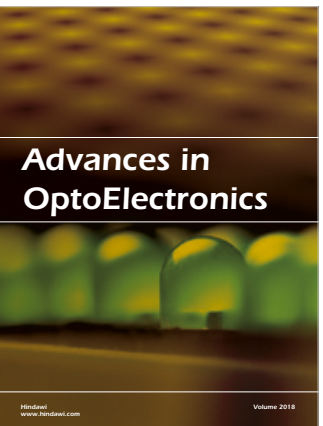

\section{Rotcting Machinery}
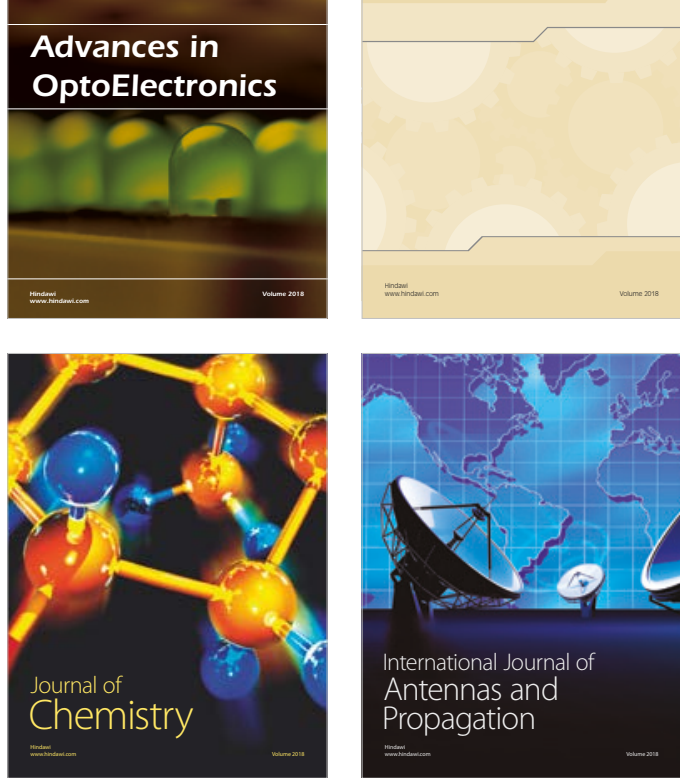

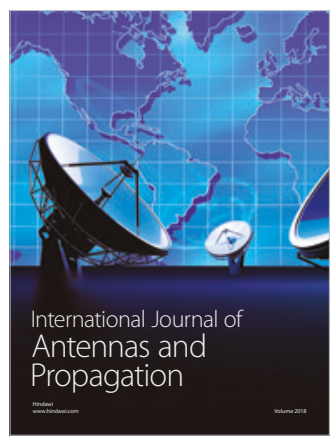

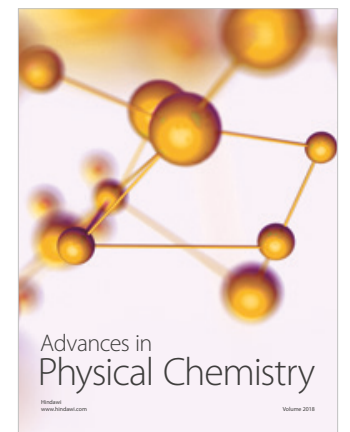

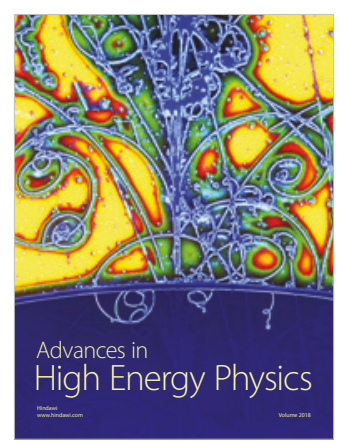

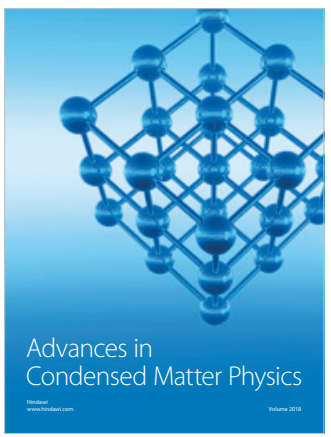

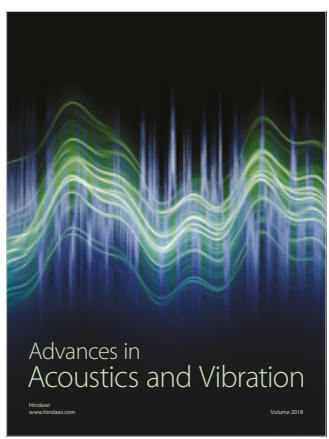

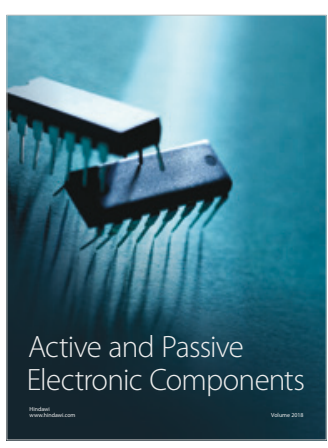
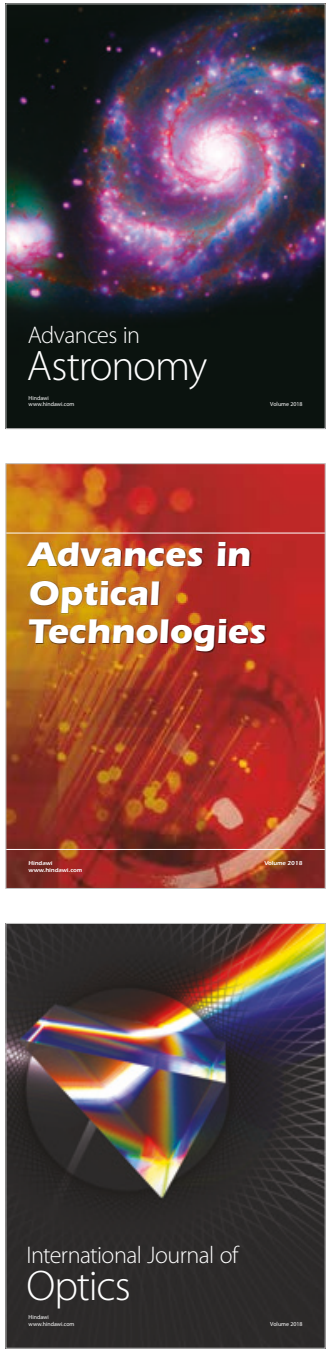